\title{
Desarrollo de habilidades de análisis crítico a partir de la identificación de errores en diagramas asociados a los efectos de las ondas sísmicas
}

DEVELOPMENT OF CRITICAL ANALYSIS SKILLS BASED ON THE IDENTIFICATION OF ERRORS IN DIAGRAMS RELLTED TO THE EFFECTS OF SEISMIC WAVES

\author{
JoSé SelLés-Martínez \\ Departamento de Ciênclas Geológicas, Faculdade de Ciências Exatas e Naturais, Universidade de Buenos Aires, Buenos Aires, Argentina. \\ E-MALL: PEPE@GL.FCEN.UBA.AR
}

\begin{abstract}
A set of $3 \mathrm{D}$ diagrams showing how seismic waves affect the materials they pass through, inducing vibrations and displacements, which contain various errors, are analyzed. The direction in which $P$ and $S$ waves reach the earth's surface, the exaggeration of shakes produced by $P$ and $S$ waves, making them comparable with those caused by Love and Rayleigh waves and assigning them equal destructive capacity, along with the fact that the mechanical properties of the materials used to build fences or power-lines and the way they deform under stresses caused by the vibrations, are some of the identified mistakes. A proposal is made to use these materials in association with conceptually correct ones in order to help students develop communicative skills transforming theoretical concepts into images and also promote critical reading of their study materials.
\end{abstract}

Resumen: Se analiza un conjunto de modelos tridimensionales que representan la forma en que las ondas sísmicas afectan los materiales que atraviesan, generando vibraciones y desplazamientos y que contienen diversos errores. Entre ellos se destaca la dirección en que las ondas P y S Ilegan a la superficie, la exageración de los desplazamientos asociados a las ondas P y S haciéndoselos comparables con los producidos por las ondas Love y Rayleigh y asignándoseles propiedades destructivas equivalentes a éstas. Tampoco se tienen en cuenta las propiedades mecánicas reales de los materiales que constituyen las cercas y tendidos eléctricos frente a la deformación inducida por las ondas ni los esfuerzos generados. Se propone utilizar estos materiales, junto con ilustraciones correctas, para que los estudiantes desarrollen habilidades tanto para la transformación de conceptos teóricos en diagramas y materiales gráficos como para el análisis crítico del material de estudio.
Citation/Citação: Sellés-Martínez, J. (2020). Desarrollo de habilidades de análisis crítico a partir de la identificación de errores en diagramas asociados a los efectos de las ondas sísmicas. Terræ Didatica, 16, 1-10, e020041. doi: 10.20396/td.v16i0.8660373.

Keywords: Body and surface waves, Ground shaking, Seismic hazard, Geoscience education.

Palabras clave: Ondas de cuerpo y superficiales, Vibraciones del suelo, Riesgo sísmico, Enseñanza de las Geociencias.

Manuscript/Manuscrito:

Received/Recebido: 08/07/2020

Revised/Corrigido: 12/09/2020

Accepted/Aceito: 30/09/2020

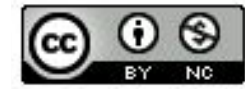

\section{Introducción}

Si bien el antiguo dicho popular advierte que "una imagen vale más que mil palabras" es importante que, para que ello se cumpla, la imagen sea correcta y no induzca a interpretaciones equivocadas. En el caso de la ciencia, y sobre todo en las ciencias de la Tierra, el uso de las imágenes es imprescindible y la dificultad para realizar esquemas didácticos muchas veces tropieza con los problemas vinculados a las diferentes escalas de tiempo y espacio en que, simultáneamente, se desarrollan la mayor parte de los procesos y eventos geológicos.

El concepto de "onda" como forma de transmisión de la energía no es sencillo y su explicación es difícil en contextos de divulgación y de educación para la prevención de los riesgos, sin embargo su comprensión es necesaria para entender por qué y cómo se mueve el terreno al paso de las ondas y cómo reaccionan las obras construidas por el hombre frente a estos impulsos. Habitualmente se utilizan en los libros de texto y artículos de divulgación esquemas diseñados para explicar los mecanismos de transmisión de los diferentes tipos de ondas a través de la materia recurriendo a dibujos en perspectiva denominados "bloques diagrama" para poder evidenciar claramente las componentes del movimiento en las tres direcciones ortogonales del espacio. Muchos de estos diagramas combinan la representación del material que constituye el suelo (simulado por un conjunto de bloques de forma prismática) con la representación de un paisaje para mostrar la relación entre los desplazamientos de las partículas del suelo y sus efectos en la superficie. Con la intención de dejar más claro cuál es el efecto de las ondas a veces se incluyen "metáforas" de los modelos análogos que no siempre son válidas. En 
esta presentación se aborda la asimilación de los cables del tendido eléctrico (o de una cerca compuesta por tablones de madera o por postes y alambres) con el resorte o la cuerda que se utilizan para simular las ondas $\mathrm{P}$ y $\mathrm{S}$, modelos análogos que, de por sí, ya poseen sus propias limitaciones (Sellés-Martínez, 2020).

En la Figura 1 se presenta un breve resumen de las ondas sísmicas que se generan tanto en el foco del sismo ( $\mathrm{P}$ y S), como en la superficie terrestre cuando es alcanzada por las primeras (Love y Raylei-
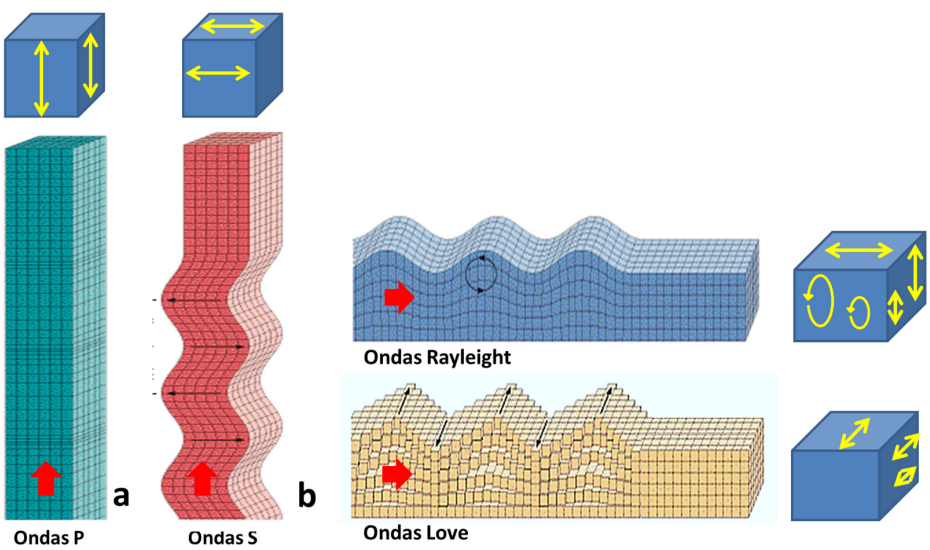

c

Figura 1. Desplazamiento de las partículas en la porción más somera del material bajo la superficie al paso de las ondas. a y b son ondas de cuerpo que viajan por el interior de la Tierra hasta llegar a la superficie. c y d son ondas de superficie, generadas en la superficie como consecuencia de la diferencia de propiedades mecánicas entre el suelo y la atmósfera. Las flechas rojas señalan la dirección de propagación de las ondas y las flechas amarillas la trayectoria de la oscilación de las partículas del suelo gh). Es importante señalar que si bien todas hacen vibrar el suelo, las que inducen desplazamientos más importantes, y por lo tanto son las que mayor potencial de daño tienen, son estas últimas.

\section{Los ejemplos encontrados}

Si bien en el desarrollo de esta contribución se utilizan como ejemplo las figuras presentadas por un sitio en particular es importante destacar que las mismas se encuentran repetidas también en otros sitios e incluso en libros muy populares (por ej. Tarbuck \& Lutgens, 2005) y existen versiones similares también muy difundidas.

Los elementos presentes en ellas que constituyen errores (o inducen a ellos) más importantes a destacar son:

a. Confusión en la dirección de propagación de las ondas P y S que llegan a la superficie

b. Confusión en la magnitud de los desplazamientos inducidos por las ondas $\mathrm{P}$ y $\mathrm{S}$ en profundidad y en superficie

c. Confusión entre el desplazamiento que provoca el terremoto sobre el plano de la falla que se activa (cuando esta tiene expresión en la superficie) con los efectos propios de las ondas

d. Desconocimiento de las propiedades mecánicas de los materiales que son afectados.

e. Desconocimiento de la importancia de los factores de amplificación y resonancia en los movimientos de los materiales superficiales del terreno y, como consecuencia, pensar que han sido producidos por la energía y el desplazamiento inducido por la onda.

Cada uno de estos puntos será comentado por separado por razones de claridad, pero en la mayoría de los casos algunos errores suelen presentarse combinados.

a. Confusión en la dirección de propagación de las ondas $P$ y $S$ que llegan a la superficie

Tal como puede verse en las Figuras $2 \mathrm{a}$ y $2 \mathrm{~b}$, se considera la dirección de propagación de estas ondas paralela al suelo. En realidad, las ondas llegan a la superficie como frentes esféricos originados en el foco, que se encuentra en profundidad y son, por lo tanto, perpendiculares o muy oblicuas al suelo, dependiendo su ángulo de incidencia tanto de la inclinación de la superficie topográfica local como de la distancia del lugar al hipocentro o foco.

En la Figura 3 puede verse un dibujo esquemático que ilustra como irradian las ondas a partir del foco y alcanzan la superficie formando un alto ángulo con la horizontal local. Se ha señalado el origen de los frentes de ondas (esferas concéntricas centradas en el hipocentro o foco y la propagación de las ondas en todas direcciones (flechas de color azul). En las interfaces entre estratos con diferentes propiedades mecánicas y, sobre todo, en la superficie terrestre, donde este contraste es máximo 


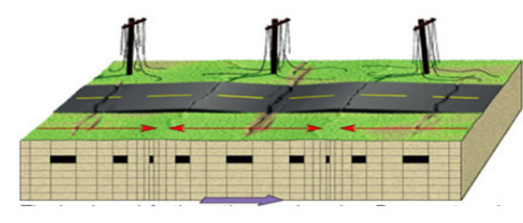

P1

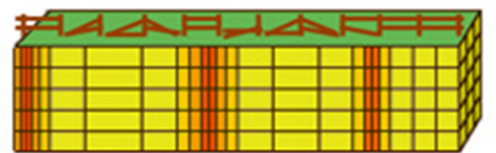

P2

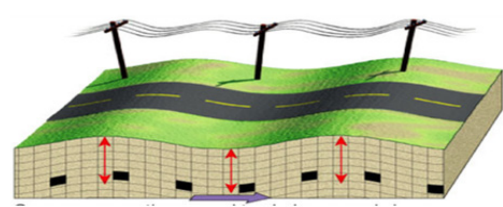

S1

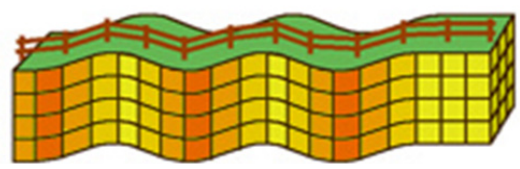

S2

Figura 2. Las ondas $\mathrm{P}$ y $\mathrm{S}$ son representadas propagándose en dirección paralela a la superficie cuando en realidad no es así sino que, como se explica en el texto y se ilustra en la figura 3 , llegan a la superficie terrestre en forma perpendicular o muy oblicua, acercándose al paralelismo sólo en regiones alejadas del foco, cuando su energía ha disminuido sensiblemente. Los esquemas P1 y S1 han sido tomados de Craven (2020); los P2 y S2 de Cires (2020)0

(suelo en contacto con aire), se generan las ondas de superficie (flechas de color rojo), que también irradian en forma concéntrica desde el epicentro hacia todos lados, pero no hacia las profundidades, ya que se atenúan rápidamente al alejarse de la superficie que motiva su aparición. Sólo se han marcado en la superficie terrestre y en el contacto entre los estratos amarillo y rosado para no sobrecargar el dibujo.

b. Confusión en la magnitud de los desplazamientos inducidos por las ondas $P$ y $S$ en profundidad y en superficie

La energía de las ondas induce una vibración de las partículas que son alcanzadas por ellas y que va transmitiéndose de una partícula a la otra. Las ondas $P$ producen un cambio en el volumen de las partículas, no producen deformación en el cuerpo que estas constituyen; las ondas S, por el contrario, no producen cambios en el volumen, pero sí inducen deformación. Tanto los cambios de volumen como los desplazamientos son imperceptibles mientras las ondas atraviesan materiales confinados, es decir que no presentan una superficie libre. Al alcanzar la superficie la energía que reciben las partículas puede hacer que las mismas sufran desplazamientos mayores al no tener otras partículas que se opongan a su movimiento. Esta situación se ejemplifica claramente con el clásico modelo consistente en un conjunto de péndulos puestos en fila de modo que todas las esferas están en contacto y, al tomar la esfera de uno de los extremos y dejarla caer para que golpeé a la siguiente, la energía es transmitida por toda la hilera (sin desplazamientos perceptibles de cada unidad) y sólo la última esfera se proyecta fuera de la fila.

c. Confusión entre el desplazamiento sobre el plano de la falla que provoca el terremoto (activación de la escarpa de falla) cuando la misma es visible en la superficie con los efectos de las ondas sísmicas

En muchos casos la ruptura del suelo es pre-existente y el último sismo solo produce la activación del desplazamiento sobre la superficie de fallas que se extienden en profundidad. Estos desplazamientos, tanto en la vertical como en la horizontal, no son resultado del paso de las ondas sísmicas, sino que se encuentran vinculados a su causa y tienen una expresión geomorfológica como resaltos en el paisaje mucho mayor que los daños causados por las ondas dado que son el resultado de la acumulación de desplazamientos producidos por numerosos terremotos a lo largo de miles e incluso millones de años. Cuando se trata del efecto

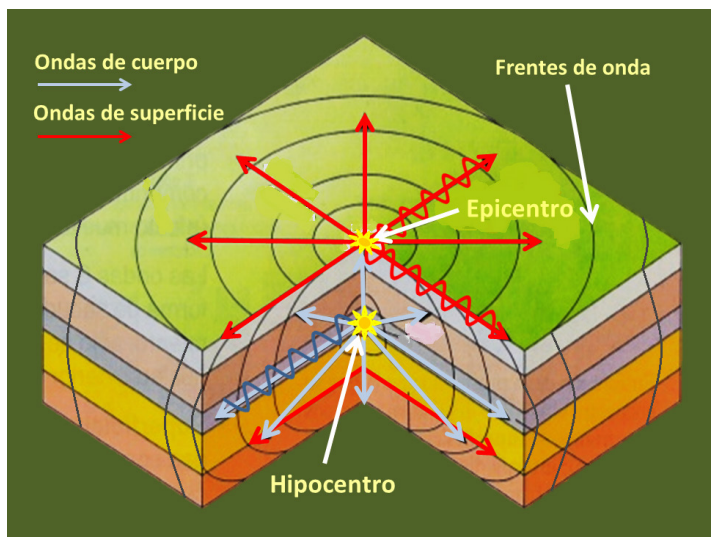

Figura 3. Bloque diagrama que muestra cómo se expanden las ondas de cuerpo y las superficiales, haciéndolo a partir del foco sísmico o hipocentro las primeras y del epicentro las segundas. Véanse los comentarios correspondientes en el texto 
del paso de las ondas la deformación del terreno es recuperada al atenuarse las vibraciones (salvo que se produzca la fracturación de los materiales superficiales al excederse su resistencia en el caso de movimientos muy violentos producidos por las ondas Love y Rayleigh) pero en el caso de la expresión superficial de los desplazamientos reemplazar por del plano de falla, la deformación es irrecuperable y el desplazamiento queda incorporado al paisaje. En https://www.youtube.com/watch?v=1vWvHaaMJnM puede verse la expresión superficial de la falla de San Andreas a lo largo de una parte de su extensa traza y los paisajes y tipos particulares de roca que la caracterizan.

\section{d. Desconocimiento de las propiedades mecánicas} de los materiales que son afectados

Los materiales (maderas, alambres, postes de alumbrado de madera, metal y hormigón, etc.) poseen propiedades mecánicas tales como dureza, flexibilidad, resistencia a la tensión, resistencia a la compresión, resistencia al corte, etc. Estas propiedades son puestas en juego cuando el material es alcanzado por las ondas y comienza a vibrar. En función de la amplitud de las ondas y de la magnitud de los desplazamientos involucrados, el material será sometido secuencialmente a esfuerzos de comprensión y tracción y también a esfuerzos de corte que pueden alcanzar el valor necesario para provocar la rotura. Las vibraciones que se mantienen por tiempos muy prolongados pueden provocar también la denominada "fatiga" del material, que cederá por la repetición de la situación de esfuerzo y no por el valor del mismo. Un material de alta resistencia a la tracción, como puede ser un alambre o un cable metálico (que pueden estirarse mucho antes de cortarse) o un material como la madera que puede deformarse mucho tanto en compresión como en tensión o corte no son los más adecuados para usar como ejemplos, y sin embargo, en la mayoría de los casos se los ha empleado para ello.

\section{e. Desconocimiento de la importancia de los factores} de amplificación y resonancia en los movimientos de los materiales superficiales del terreno y, como consecuencia, pensar que han sido producidos por las ondas mismas

En muchos casos se ilustran los efectos del sismo con imágenes de la rotura del suelo (Fig. 4a) o de la deformación inducida en las vías del ferrocarril (Fig. 4b). Sin embargo, estos daños no son resultado inmediato y exclusivo de las ondas que afectan

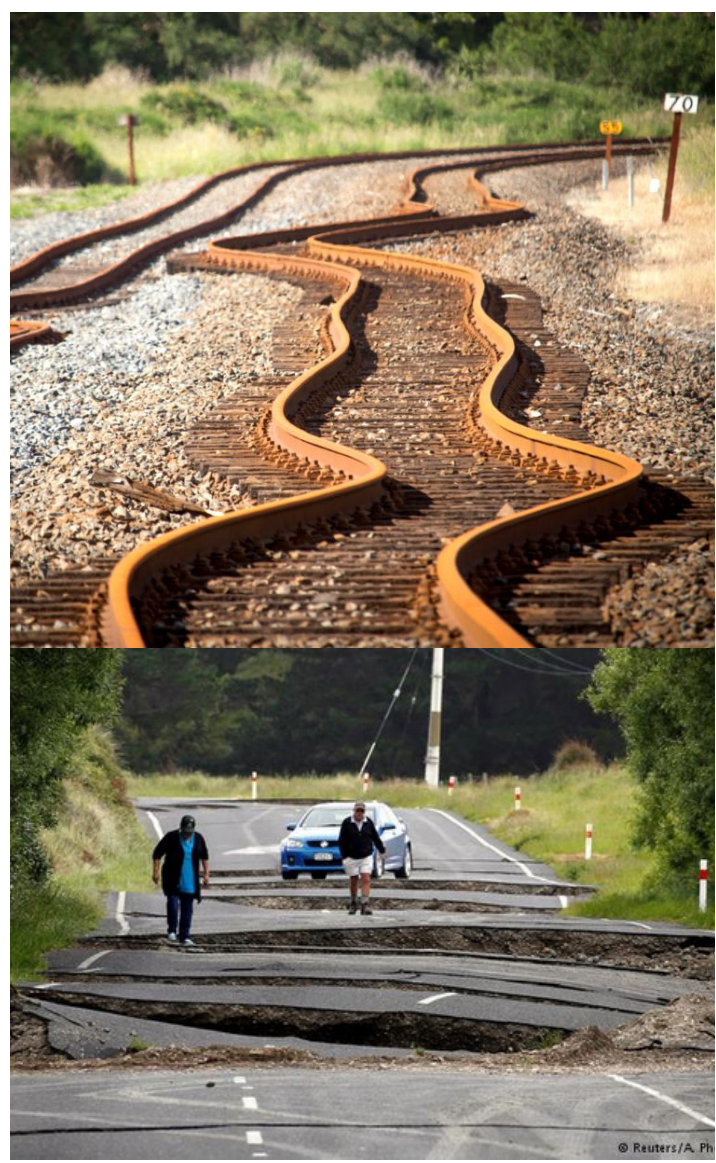

Figura 4. Figura 4. Deformación y rotura resultantes de las ondas superficiales de tipo Love. Obsérvese que en ambos casos el terreno bajo las estructuras carece de cohesión lo que induce la amplificación de los desplazamientos. a) Vías del ferrocarril en Nueva Zelanda, las durmientes están asentadas sobre balasto y las vías son fácilmente impulsadas hacia los lados y deformadas por el movimiento ondulante lateral del terreno durante el sismo de Kaikoura (14 de Noviembre de 2016). Imagen tomada de Extreme Pursuit (2020). b) Fracturación y desplazamiento de losas de pavimento durante ese mismo evento. El suelo poco cohesivo situado bajo la carpeta asfáltica amplifica las ondulaciones del terreno. Las fracturas posiblemente se localicen en las zonas de unión de los bloques de concreto. Imagen tomada de The Storypedia (2020)

el terreno sino que, en condiciones particulares, la amplitud de las vibraciones es amplificada por diversas causas (coincidencia de la frecuencia de las ondas que llegan con la frecuencia de vibración natural de los cuerpos, existencia de suelos sin ningún tipo de cohesión, fenómenos de fluidización de sedimentos saturados en líquidos y desestabilización de los cimientos de los edificios, aludes provocados por la fluidización de suelos, etc.).

\begin{tabular}{c|c|c|c|c|c}
\hline (C) Terrae Didat. & Campinas, SP & v.16 & $1-10$ & $\mathrm{e} 020041$ & 2020 \\
\hline
\end{tabular}




\section{Análisis de las ilustraciones problemáticas}

En el esquema de la Figura 2 se han ejemplificado los efectos del movimiento del suelo al paso de las ondas P y S representando los daños producidos por las mismas sobre una cerca de madera y sobre los cables de un tendido eléctrico. Dejando de lado que el esquema asume que estas ondas se propagan en dirección paralela al suelo (ya se ha señalado que ello es improbable), el mismo contiene otras inexactitudes.

Para el caso de las ondas P (esquemas P1 y P2) daños de esa magnitud no son posibles en función exclusiva de los desplazamientos del terreno debido a estas ondas, ya que estos no tienen carácter macroscópico. Debe tenerse en cuenta además que las cercas están hechas de madera, un material que absorbe deformaciones en forma elástica y no debería romperse ante esfuerzos débiles (si así ocurriera se produciría una contradicción, ya que su uso está recomendado para las construcciones sismoresistentes). Puede destacarse también que, en general, son mucho más frecuentes los fuertes vientos que los fuertes sismos y es muy excepcional que se informen cortes de cables por efecto del viento. Sin embargo, los efectos de amplificación de la amplitud en función del alto de los postes y su frecuencia de vibración puede hacerlos oscilar visiblemente, pero sin que lleguen a cortarse los cables, como puede verse en algunas de las escenas que fueron grabadas por la población en ocasión del terremoto de Oaxaca del 23 de Junio del 2020, que tuvo una magnitud 7,4 (por ejemplo en https:// www.youtube.com/watch? $\mathrm{v}=\mathrm{TO} 6 \mathrm{WlN} 3 \mathrm{fZ}$ tw y en https://www.youtube.com/watch?v=De5ST$13 \mathrm{MHwY})$. Los cables eléctricos, por su parte, no se colocan tensos entre los postes sino que los mismos "cuelgan", dibujando una catenaria que es calculada en función de su peso y su resistencia a la tracción, por lo que su longitud es mayor que la distancia que separa a los postes que los sostienen. Esto les permite soportar el empuje del viento sin que los esfuerzos de tracción generados produzcan la rotura y muy difícilmente se llegue a la situación que ilustra el esquema en cuestión.

Para el caso de las ondas S (esquemas S1 y S2) los cambios en la distancia ente los puntos de fijación de los tramos de cable serían mucho mayores que para la onda $\mathrm{P}$, por lo que su posibilidad de cortarse sería mayor que en el caso anterior, sin embargo el dibujo sólo sugiere que ondulan. En el caso de la cerca ocurre algo similar, la deforma- ción es mucho más intensa (imagen S2) ya que los rectángulos originales son transformados en trapecios, lo que implica la rotación de los listones con respecto a los puntos de fijación y, esta situación es mucho más difícil de soportar, dado que la deformación se concentra en estos nodos y puede fracturar los listones en esos puntos o por lo menos sacarlos de sus encastres si no están fijados con clavos o tornillos.

El esquema que se presenta en la Figura 5 presenta también el problema de haberse asumido una onda P desplazándose en dirección paralela al suelo y se han sobredimensionado los desplazamientos horizontales y no se han tenido en cuenta las propiedades mecánicas de los materiales utilizados. Un problema adicional es que el diagrama (al ser estático y mostrar los alambres rotos colgando y no sacudiéndose en el aire) parece representar el resultado final del pase de la onda y, los cambios representados en la distancia entre los postes (originalmente equidistantes) sugieren que algunos sectores del terreno han quedado comprimidos y otros se han extendido en forma permanente, cuando de ningún modo es así. Debe señalarse, incluso, que si los alambres se rompen como resultado de la extensión durante la fase expansiva y esta fase afecta sucesivamente a todos los puntos del terreno la paso de la onda, como resultado final todos los alambres deberían haberse cortado...
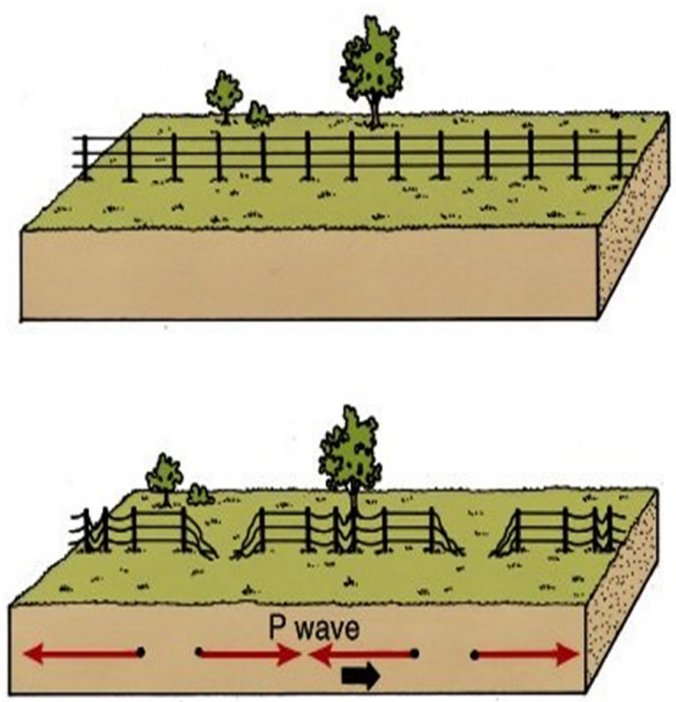

Figura 5: El paso de las ondas $\mathrm{P}$ no cambia la distancia entre puntos del terreno en la magnitud que la figura sugiere. Véase la discusión en el texto. Esquemas tomados de Tiwana (2020) 
En la Figura 6 se ilustran otros dos esquemas que, además de presentar las ondas con trayectoria horizontal, también conducen a equívoco porque no tienen en cuenta la totalidad de la situación al producirse el paso de la onda. En la columna de la izquierda, si bien los desplazamientos representados son muy amplios, en este caso serían posibles pues se trata de una onda superficial (onda Love); pero puede verse que el diseñador, que cortó esos mismos cables para desplazamientos mínimos en el esquema P1 de la Figura 3, no los corta ahora que los desplazamientos son francamente importantes, como demuestra el cambio de longitud entre los puntos a y b al pasar a la posición $\mathrm{a}^{\prime}$ y b' durante el paso de la onda. En la columna de la derecha, por su parte se han propuesto desplazamientos que son absolutamente inaceptables para una onda $\mathrm{S} y$ la figura no aclara que el plano de polarización de la onda es horizontal (habitualmente se representan ondas $\mathrm{S}$ polarizadas verticalmente) y tampoco tiene en cuenta el cambio de longitud de la cerca que, al pasar de la forma recta a la ondulada, multiplica su longitud por un factor aproximado a 1.5. Si se toma el segmento de la distancia entre los puntos a y b (antes de la deformación) con sus homólogos $a^{\prime} y^{\prime} b^{\prime}$ (durante el paso de la onda) se ve que la distancia entre ellos aumenta considerablemente y ni el terreno ni la cerca sufren daño alguno...
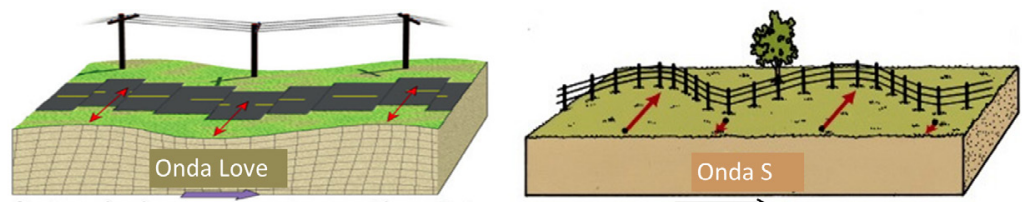

Esquemas tridimensionales ilustrando el paso de las ondas Love y $S$
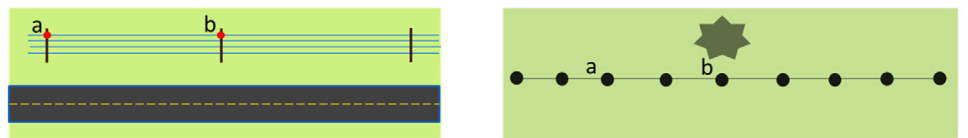

Vista en planta del terreno antes del paso de la onda en cada esquema
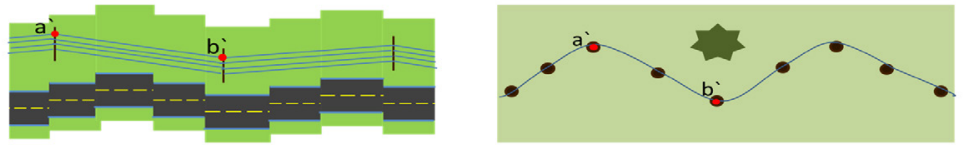

Vista en planta durante el paso de la onda Love en cada esquema

Figura 6: En la parte superior se reproducen los esquemas analizados tal cual han sido publicados. En la parte inferior se han reconstruido para su comparación los equivalentes en planta para una situación inicial de reposo y al momento del paso de las ondas que ilustran los diagramas tridimensionales. Véase la explicación en el texto. Esquemas de la parte superior tomados de Craven, 2020 y Tiwana, 2020. Las vistas en planta son interpretaciones de los mismos realizadas por el autor para ilustrar la explicación que se desarrolla en el texto

\section{Actividad didáctica sugerida} repetidos, no agota su consideración. Haberlos identificado no constituye un mérito, sino que es fruto del desarrollo de una habilidad y del hábito de ponerla en práctica y son esta habilidad y este hábito lo que es deseable que adquieran los estudiantes, para aplicarlo con el objetivo de desarrollar las habilidades n cionadas puede ser lo más interesante que hacer con que se la desarrollará: b. Segunda parte. Abordar el análisis de los esqueesquemas utilizados en la Primera parte es coherente con los diseños que han realizado los dibujantes.

A partir del esquema de la Figura 8 a puede introducirse una actividad adicional que permitirá verificar otro error de dibujo. Si se observa el detalle en la Figura 9, puede verse que la distancia entre la mano y el punto de fijación de la cuerda en la pared no aumenta. Sin embargo, es imposible introducir la perturbación en la cuerda sin modificar la posición de la mano, como los alumnos podrán verificar si intentan hacer la experiencia. En Sellés-Martínez (2020) se ha indicado esta situación como una limitación seria del modelo de la cuerda para la ejemplificación de la propagación de las ondas S. 
Figura 7. Esquemas que representan los movimientos causados por la propagación de las ondas en los materiales que atraviesan. Nótese que las ondas $\mathrm{P}$ y $\mathrm{S}$ han sido dibujadas propagándose en dirección vertical. Fuente: cK12 (2020). Traducción del autor
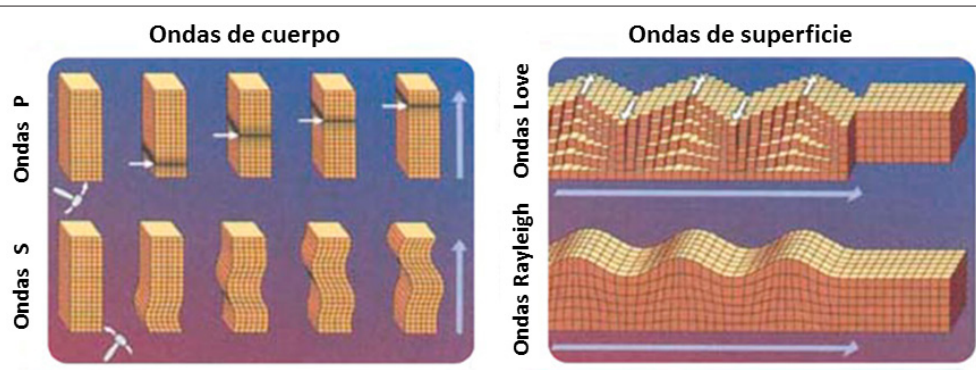

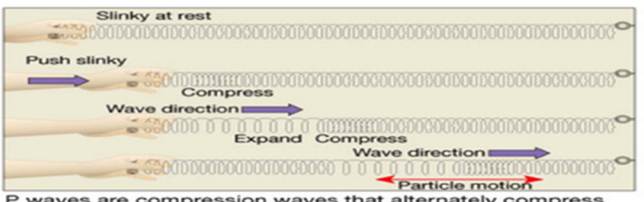

P waves are compression waves that arternate motion
and expand the material through which

a

b

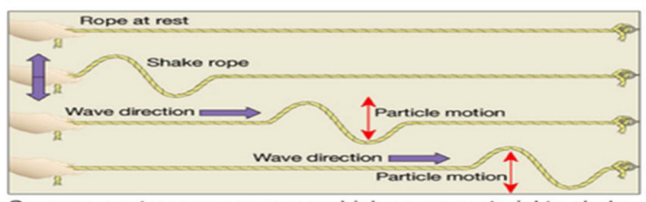

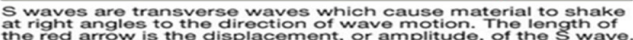

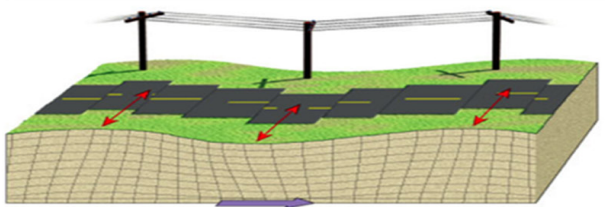

One type of surface wave moves the ground from side to
side and can damage the foundations of buildings.

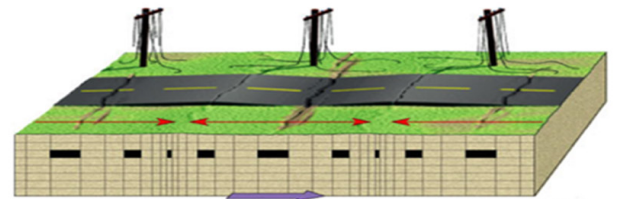

The back-and-forth motion produced as P waves travel
along the surface can cause the ground to buckle and fracture.

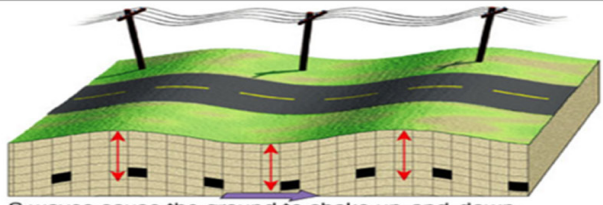

S waves cause the ground to shake up-and-down

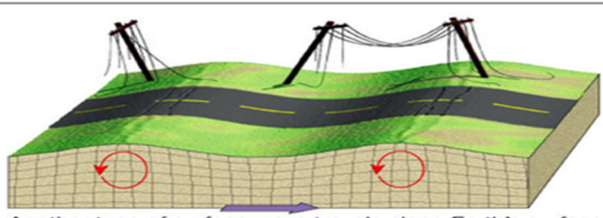

Another type of surface wave travels along Earth's surface much like rolling coean waves. The arrows show the follows

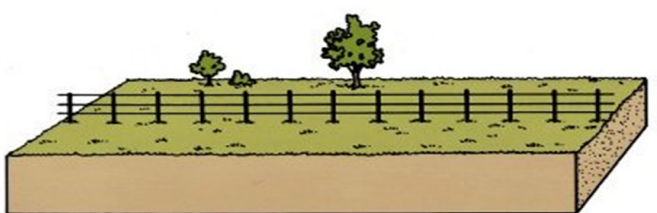

(A)

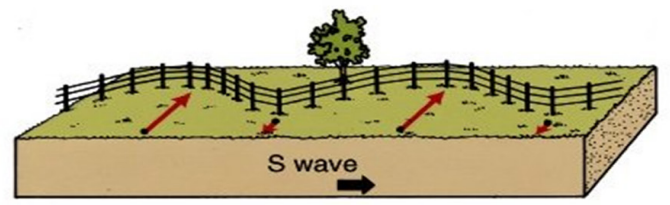

(C)

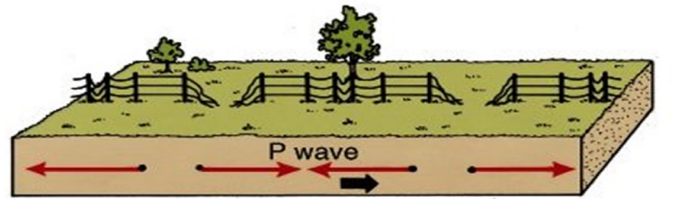

(B)

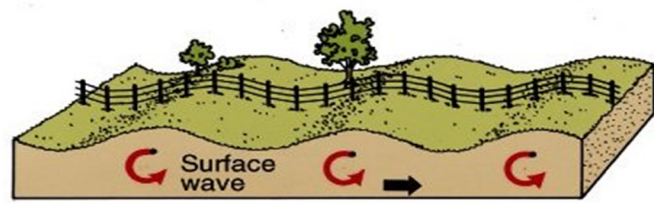

(D)
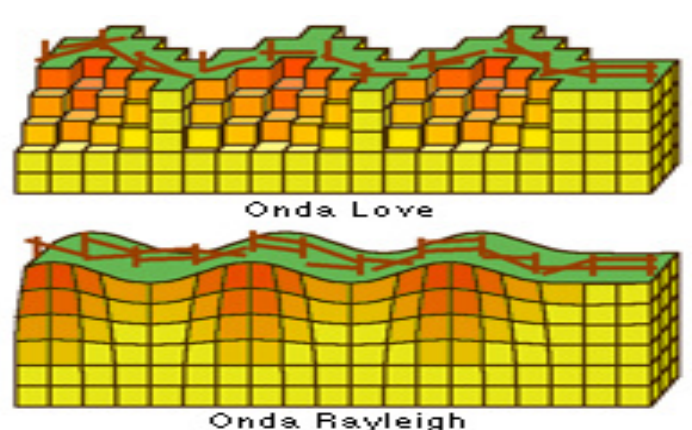

Figura 8. Esquemas que han dado origen a este trabajo y que los alumnos pueden analizar críticamente como parte de su entrenamiento. Fuentes: a) Craven (2020), b) Tiwana (2020), c) Cires (2020) 


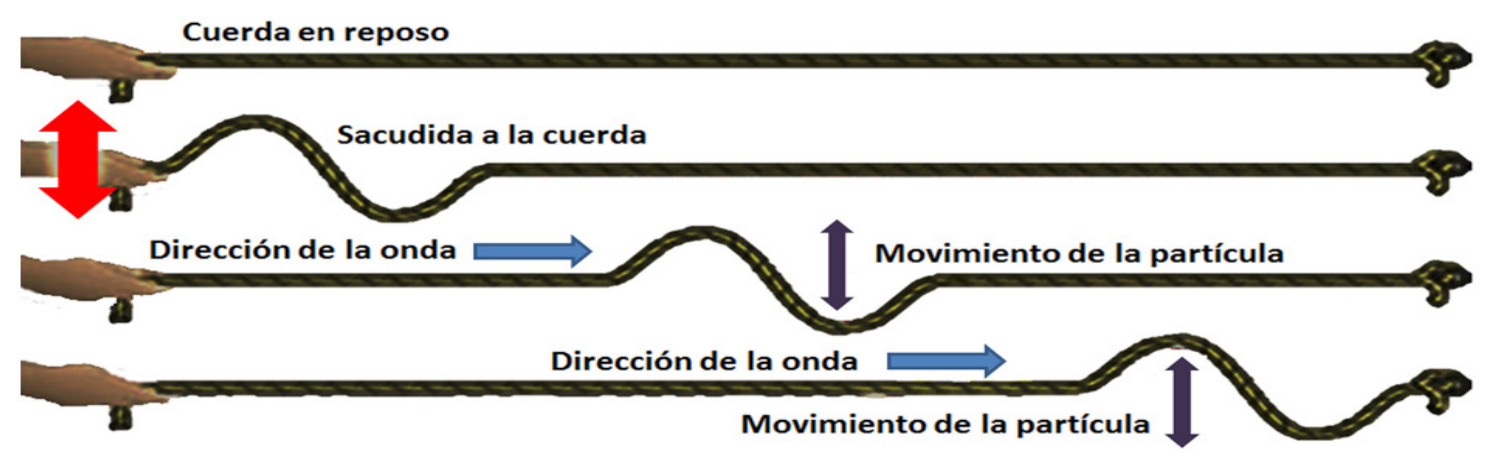

Figura 9. Es imposible introducir las ondulaciones en la cuerda sin reducir la distancia entre la mano y la pared. Esquema tomado de Craven (2020), traducido y adaptado por el autor

c. Tercera parte. Como una forma de cerrar la propuesta y fijar los conceptos se puede pedir a los alumnos que realicen sus propios esquemas. Puede pedírseles que hagan una búsqueda en la Internet de los valores esperables para los desplazamientos causados por las ondas de cuerpo en la superficie y los que resultan de las ondas de superficie. La revisión de las propuestas puede hacerse en forma grupal y, para cerrar la discusión se comparan los dibujos de los alumnos con la Figura 10, considerada correcta. Pueden exhibirse algunas fotografías del tipo ilustrado en la Figura 4, que muestran importantes fracturas en el terreno y deformación de vías de ferrocarril para que analicen qué ondas pueden haberlas producido.
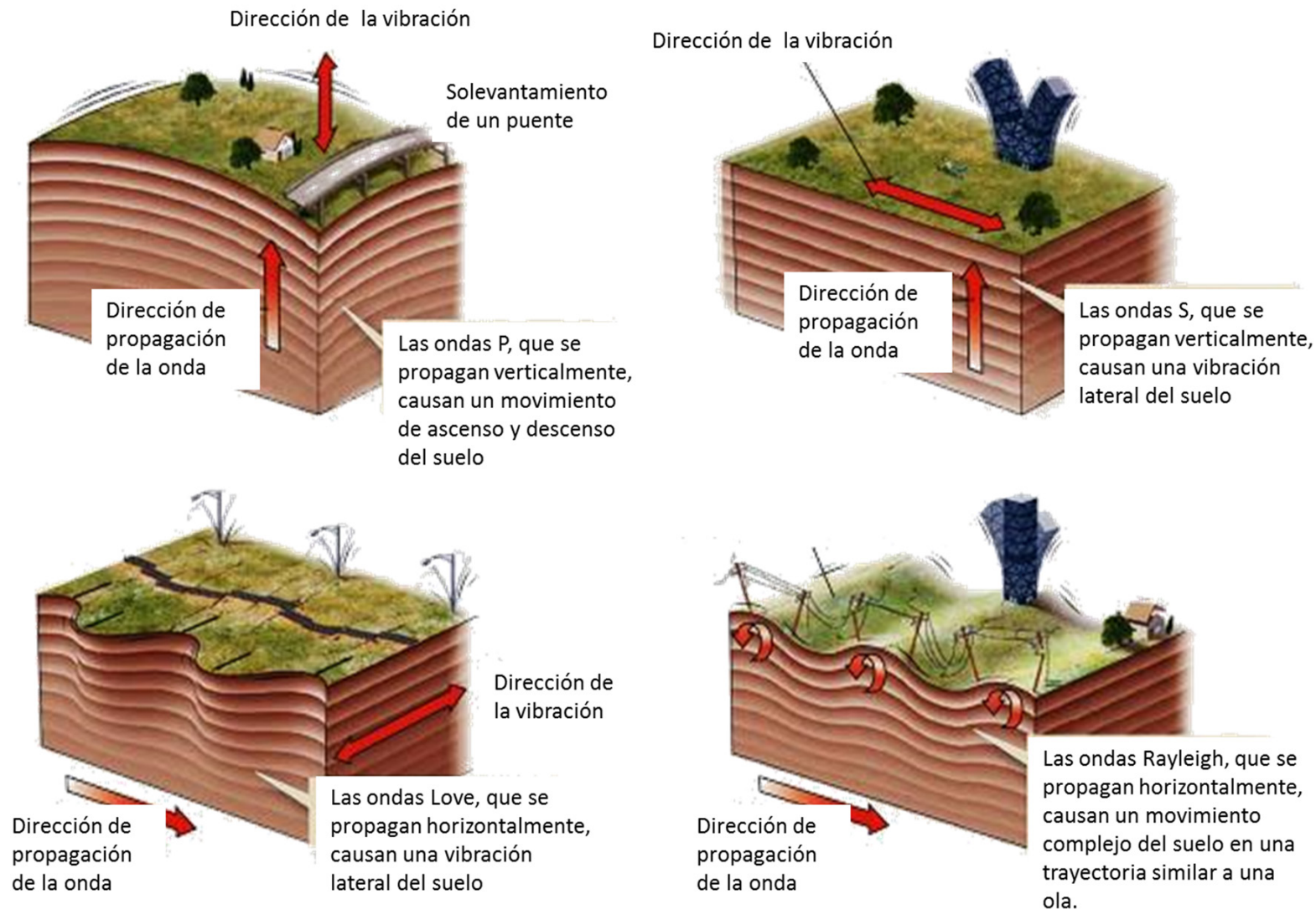

Figura 10. Esquemas que ilustran los efectos de las ondas sísmicas sobre el terreno y que no contienen los errores señalados en esta presentación. Fuente: O.K. (2020). Traducción y adaptación del autor 


\section{Reflexiones finales}

Los problemas identificados en las figuras analizadas, que claramente redundan en la introducción de errores en la comprensión y aprendizaje del tema por parte de los estudiantes, pueden sintetizarse en los siguientes puntos:

- Focalización del problema en lo que ocurre en una dirección en particular y, como consecuencia, surgimiento de problemas en la percepción simultánea de lo que ocurre en las otras dos dimensiones.

- No mantenimiento de las relaciones de escala entre la amplitud de las vibraciones reales y su representación en los diagramas. Esto es algo que los no expertos no pueden identificar por si solos y que debería ser destacado y advertido cada vez que se distorsionan las relaciones reales.

- Confusión entre los desplazamientos causados por el paso de las ondas con la ruptura y desplazamiento causados por los terremotos poco profundos a lo largo de fracturas en la superficie terrestre.

- Utilización de diagramas a escala del paisaje (decenas de metros) para ilustrar vibraciones que tienen lugar a escala de las partículas o a lo sumo de algunos centímetros.

Se recomienda que para la representación gráfica de situaciones de alteración de una posición de

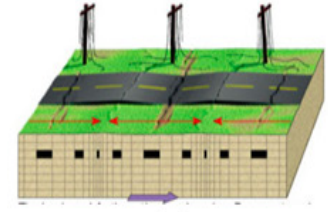

(a)

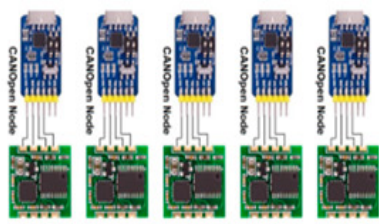

(e)

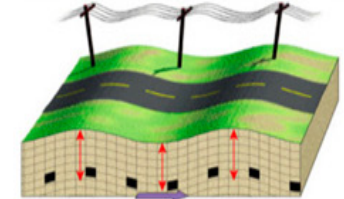

(b)

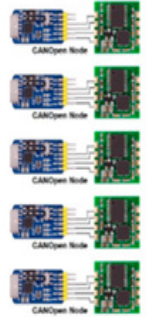

(f)

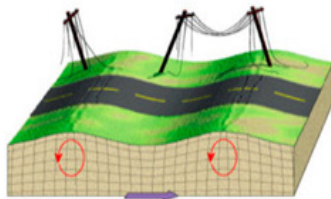

(c)

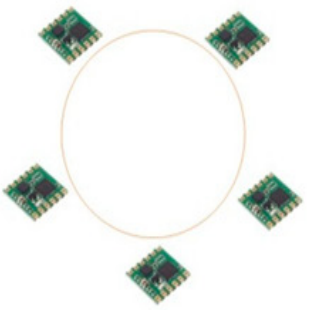

(g)

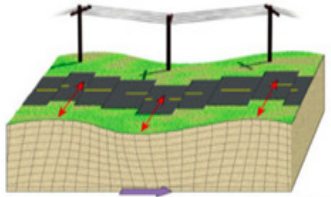

(d)

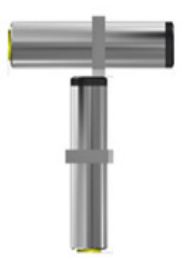

(h)

Figura 11. Empleo de un esquema equivocado de dirección de transmisión de las ondas P y S en el diseño de un sistema de alarma temprana. Fuente: Tariq et al. (2020) 


\section{Referencias}

Cires, A. C. (2020). Centro de Instrumentación y Registro Sísmico, Sociedad Civil. URL: https://blogcires.files.wordpress.com/2014/06/ondas.jpg Acceso 15.09.2020.https://blogcires.files.wordpress. com/2014/06/ondas.jpg. Acceso 15.09.2020.

cK12 (2020). Seismic Waves. URL: https://www.ck12. org/earth-science/Seismic-Waves/lesson/SeismicWaves-HS-ES/. Acceso 15.09.2020.

Craven, H. (2020). Christchurch Earthquaques. URL: https://christchurchearthquakes-hcraven.weebly. com/seismic-waves.html

Extreme Pursuit. (2020). Kaikoura Earthquake Report. URL: https://extreme-pursuit.com/kaikouraearthquake-report/. Acceso 15.09.2020.

O.K. (2020). Learning Geology, How do earthquakes cause damage. URL: http://geologylearn.blogspot. com/2015/11/how-do-earthquakes-causesdamage.html. Acceso 15.09.2020.

Sellés-Martínez, J. (2010). Aprender de los errores... ajenos. Algunos casos muy ilustrativos para desarrollar en el aula. Simposio de Enseñanza de la Geología. XIX Congreso Geológico Argentino. Asociación Geológica Argentina. Publicación Especial Serie D $\mathrm{N}^{\mathrm{O}} 13, \mathrm{p}$ 62-75.

Sellés-Martínez, J. (2012). Ciencias de la tierra: errores habituales en las publicaciones de divulgación y medios de información, sus causas y su interés y aplicaciones en la enseñanza. Parte 2: ilustraciones erróneas. Comunicaciones del XVII Simposio de Enseñanza de la Geología. Universidad de Huelva. p 251-256.
Sellés-Martínez, J. (2020). Aciertos y falencias de los modelos analógicos asociados a terremotos utilizados en enseñanza. Terre Didatica, 16, 1-11, e020029. doi: https://doi.org/10.20396/td.v16i0. 8658592.

Sellés-Martínez, J. (2007). Misleading conceptions lead to the belief that the Mantle is liquid. Geographie und ihre Didaktik, 35(4), 207-217.

Sellés-Martínez, J. (2010). Ciencias de la Tierra: errores habituales en las publicaciones de divulgación y medios de información. Sus causas y su interés y aplicaciones en la enseñanza. Parte 1: Errores de traducción. Comunicaciones del XVI Simposio de Enseñanza de la Geología, (Teruel, España), p 243-250.

Tarbuck, E. J.\& Lutgens, F. K. (2005) Ciencias de la Tierra: una introducción a la geología física. PearsonPrentice Hall Eds. 686p.

Tariq, J., Touati, F., Al-Hitmi, J. A. E., Crescini, D. \& Ben Mnaouer, A. (2019). A Real-Time Early Warning Seismic Event Detection Algorithm Using Smart Geo-Spatial Bi-Axial Inclinometer Nodes for Industry 4.0 Applications. Applied Sciences, 9(18), 3650; https://doi.org/10.3390/app9183650

The Storypedia. . (2020). 7.8 Magnitude Earthquake Hits New Zealand And Creates Tsunami Along The East Coast. URL: http://www.thestorypedia.com/ news/7-8-magnitude-earthquake-hits-newzealand-and-creates-tsunami-along-the-eastcoast/. Acceso 15.09.2020.

Tiwana, J. K. (2020). Seismic waves. URL: https://www. slideshare.net/jktiwana/seismic-waves-45183166. Acceso 15.09.2020. 